\title{
The Effect of Ultraviolet Radiation on Ultrasonic Properties of Polyurea
}

\author{
Ian Whitten $^{1}$ and George Youssef ${ }^{1,2,3}$ \\ ${ }^{1}$ California State University Northridge, Mechanical Engineering Department \\ ${ }^{2}$ San Diego State University, Mechanical Engineering Department
}

\begin{abstract}
Polyurea is used in military and civilian applications where exposure to the sun is commonly of long duration, which can deteriorate its mechanical performance to suboptimal levels. This study reports on the ultrasonic properties of polyurea as a function of ultraviolet radiation exposure duration and temperature. Six sets of samples were continuously exposed to ultraviolet radiation for different durations up to 15 weeks. Control samples were also tested that did not receive UV exposure. The ultrasonic properties were measured using high-frequency contact ultrasound transducers in pitch-catch configuration. All exposed samples exhibited significant color changes from transparent yellow to opaque light brown. Changes of color were observed as early as 3 weeks of UV exposure. Nonetheless, crazing was only observed in samples of $6,9,12$, and 15 weeks of continuous exposure. The size of the surface cracks increased as the duration of exposure continued. The effect of extended UV radiation on the acoustic properties was noted to be minimal, where elastic and shear moduli decreased monotonically after an initial increase during the first 3 weeks of exposure. The attenuation properties were measured at $23^{\circ} \mathrm{C}$ and $40^{\circ} \mathrm{C}$. The p-wave attenuation did not show any notable change, while the s-wave attenuation monotonically decreased as the temperature and UV exposure duration increased.
\end{abstract}

Keywords: Polyurea; Artificial weathering; Ultraviolet radiation; Ultrasonic properties.

${ }^{3}$ Corresponding author: Tel.: +1- 619-594-6649. Fax: +1-619-594-3599. E-mail: gyoussef@ mail.sdsu.edu 


\section{Introduction}

Polyurea is a thermoset elastomer which, due to its superior mechanical and physical properties, impact mitigation, moisture and chemical resistance, long elongation at tearing and a wide range of operating temperatures, makes it a suitable candidate for many engineering applications (Roland, et al., 2007; Heinrich \& Kaliske, 1997; Klüppel \& Heinrich, 2000; Persson, et al., 2005; Bond, Morton \& Krol, 1984; Rahalkar, 1989; Hoppie, 1982; Choi, Roland \& Bissonnette, 1994; Davidson et al., 2004; Barsoum, G.S. \& Dudt, P.J., 2010; Amirkhizi, et al., 2006; Amini, Isaacs \& Nemat-Nasser, 2009; Jain, 2007; Kim, 2008; Youssef, 2011). For example, polyurea adhesive is used in joint construction for civilian and military applications (Jain \& Gupta, 2012; Jain, Gupta \& Basu, 2005; Jain, Youssef, \& Gupta, 2012; Kim, et al., 2012). These joints, and structures in general, are exposed to impact loading with different amplitudes and rates. The superior impact mitigation properties of polyurea over a wide range of frequencies makes it the ideal candidate for such applications (Barsoum, G.S. \& Dudt, P.J., 2010; Amirkhizi, et al., 2006; Amini, Isaacs \& Nemat-Nasser, 2009; Jain, 2007; Kim, 2008; Youssef, 2011; Jain \& Gupta, 2012; Jain, Gupta \& Basu, 2005; Jain, Youssef, \& Gupta, 2012; Kim, et al., 2012; Youssef \& Gupta, 2012; Youssef \& Gupta, 2013; Zhao, Knauss, \& Ravichandran, 2007; Knauss, \& Zhao, 2007; Sarva, et al., 2007; Jiao, Clifton, \& Grunschel, 2006; Amini, Isaacs, \& Nemat-Nasser, 2010; Gupta, \& Youssef, 2014), as does its moisture resistance, especially for joints exposed or submerged in water (Kim, 2008; Kim, et al., 2012). Other applications that make use of the superior moisture resistance properties of polyurea include water well walls and roof protection. These applications suggest different environments where polyurea is expected to operate, with most polyurea exposed to harsh natural environmental effects (weathering) such as ultra-violet radiation, change in temperature, and humidity for extended durations and different ranges. Although polyurea has been extensively studied at different strain rates and levels (Roland, et al., 2007; Heinrich \& Kaliske, 1997; Klüppel \& Heinrich, 2000; Persson, et al., 2005; Bond, Morton \& Krol, 1984; Rahalkar, 1989; Hoppie, 1982; Choi, Roland \& Bissonnette, 1994; Davidson et al., 2004; Barsoum, G.S. \& Dudt, P.J., 2010; Amirkhizi, et al., 2006; Amini, Isaacs \& NematNasser, 2009; Jain, 2007; Kim, 2008; Youssef, 2011; Jain \& Gupta, 2012; Jain, Gupta \& Basu, 2005; Jain, Youssef, \& Gupta, 2012; Kim, et al., 2012; Youssef \& Gupta, 2012; Youssef \& Gupta, 2013; Zhao, Knauss, \& Ravichandran, 2007; Knauss, \& Zhao, 2007; Sarva, et al., 2007; Jiao, Clifton, \& Grunschel, 2006; Amini, Isaacs, \& Nemat-Nasser, 2010; Gupta, \& Youssef, 2014; Qiao, et al., 2011; Zhao, Knauss, \& Ravichandran, 2009), it is yet to be thoroughly investigated for the effect of weathering conditions, specifically ultra-violet radiation.

Jain, Youssef and Gupta (2012) examined the use of polyurea in joint construction to enhance the interfacial strength and fracture toughness of E-glass composite/polyurea/steel. They reported a dramatic increase in the joint fracture toughness. Gupta et al. also investigated the hydrothermal effects on the response of polyurea at high strain rate (Kim, 2008; Kim, et al., 2012). Additionally, they investigated the effect of chemistry change on the variation of intrinsic 
properties of polyurea-based joints and reported the dynamic fracture toughness and tensile strength of these joints (Jain \& Gupta, 2012; Jain, Gupta \& Basu, 2005; Jain, Youssef, \& Gupta, 2012; Kim, et al., 2012). Youssef et al. have recently reported on the dynamic mechanical properties of polyurea when loaded under high strain rates using laser induced stress waves (Youssef, 2011; Youssef \& Gupta, 2013). They reported on the constitutive stress-strain relationship of polyurea loaded at high strain $\left(\sim 10^{5} \mathrm{~s}^{-1}\right)$. They also reported the intrinsic tensile strength of polyurea to be $93.1 \pm 5 \mathrm{MPa}$ at an ultra-high strain rate of $1.67 \times 10^{7} \mathrm{~s}^{-1}$. Finally, Youssef et al. (2012) reported an unusual resonance phenomenon in which transmitted stress wave amplitudes almost 16 times the incident stress wave amplitudes were recorded when polyurea layers (0.07 mm-3.25 mm thick) sandwiched on both sides by the same acrylic and polycarbonate plates of approximately $1.5 \mathrm{~mm}$ thickness were subjected to laser-generated stress waves of several nanoseconds in duration.

Clifton et al. have also investigated dynamic properties of polyurea at high strain rate under pressure and pressure/shear combined loading using an impact plate experimental procedure (Jiao, Clifton, \& Grunschel, 2006). They reported on the pressure-shear dependency of polyurea deformation and found the spall strength of a thin polyurea sheet to be $101 \mathrm{MP}$, which agrees well with Youssef et al. results stated above. The research teams of Knauss et al. and Nemat-Nasser et al., independently investigated the dynamic behavior of polyurea under moderate strain rates using a split Hopkinson pressure bar (SHPB) experimental setup (Amirkhizi, et al., 2006; Amini, Isaacs \& Nemat-Nasser, 2009; Zhao, Knauss, \& Ravichandran, 2007; Knauss, \& Zhao, 2007). They established an experimentally based linearly viscoelastic model. However, Nemat-Nasser's group included the effect of change in temperature during the deformation mechanisms in the constitutive linearly viscoelastic model, while Knauss's group only reported on the time-temperature superposition principle as it applies to polyurea's master curve. Boyce et al. and Roland et al. investigated its behavior at moderate and low strain rate (Roland, et al., 2007; Sarva, et al., 2007) and they compared the behavior of polyurea in tension and compression conditions, finding that deformation is sensitive to polyurea chemistry. Recently, Nemat-Nasser et al. reported on the dynamic and ultrasonic properties of polyurea (Qiao, et al., 2011). Knauss et al. have also studied the combined pressure-shear loading on polyurea samples loaded under low-strain rates (Zhao, Knauss, \& Ravichandran, 2009). Despite 
this extensive research done on polyurea, the effect of ultra-violet radiation on the mechanical performance of polyurea has not yet been reported. It is crucial to understand the long-term reliability of polyurea especially for applications where sun exposure is expected.

The purpose of this paper is to report the effect of the extended exposure to ultraviolet radiation on the acoustic properties of polyurea. Samples were continuously exposed to UV radiation for up to 15 weeks to simulate extended exposure to sun light, which is expected in normal operating conditions where polyurea is deployed in the field. All samples were exposed at a rate of $164 \mathrm{~J} / \mathrm{day}$. The acoustic properties, i.e. the pressure and shear wave speeds as well as the corresponding attenuation coefficients, were measured using single element piezoelectric ultrasound transducers with a central frequency of $2.25 \mathrm{MHz}$. Details of the sample construction procedure and experimental protocol are discussed in the next section. The results from this investigation can be used to determine the overall reliability of polyurea-based protective armors after certain sunlight exposure duration.

\section{Materials and methods}

Polyurea sheets were prepared by mixing oligomeric diamine (AirProduct Versalink P-1000) and a diphenylmethane diisocyanate prepolymer (DOW Isocynate 143-L) with ratio of 4:1 by weight, which translates to $1: 1$ by molecular weight. The mixture was slowly poured into a Tefloncoated $1 \mathrm{~mm}$ deep aluminum mold from a height of approximately 1 meter to limit the formations of bubbles by allowing entrapped air to escape from the poured mixture before conforming to the mold surface. The molds were machined in-house and coated with non-stick Teflon spray after each use to ease the removal of cured sheets from the molds. The mixture was then cured in a vacuum oven (VWR Scientific1430) at $80 \pm 1^{\circ} \mathrm{C}$ for 24 hours, after which the sheets were noted to be fully cured (Gupta and Youssef 2014). The cured sheets of polyurea were bubble-free except around the peripherals of the sheet, which was avoided during diecutting of the samples used in the experiment.

For the ultrasound measurements, 18 discs of $25 \mathrm{~mm}$ diameter were cut from a single polyurea sheet with nominal thickness of $1 \mathrm{~mm}$. The thickness of $1 \mathrm{~mm}$ was based on polyurea's high shear attenuation, where the shear attenuation prohibited measuring the shear wave 
characteristics in thicker samples. The samples were divided into six sets and each set consisted of three specimens. Five sets were placed into UV radiation exposure system, while one set was kept unexposed to measure the acoustic properties of unexposed polyurea. The UV system (UVDOCTOR DDU2024) consisted of two UV bulbs with a peak wavelength of 360nm and two other bulbs with a peak wavelength of $420 \mathrm{~nm}$. The system and the samples within were enclosed by mirror-finished stainless steel sheets to create a chamber where ultraviolet radiation is always reflected onto the samples. The first set of samples was removed after three weeks, at which the first instance of noticeable change in the color of polyurea was observed. The remaining sets were removed at an increment of three weeks from the preceding set, where the last set was removed from the exposure system after 15 weeks of continuous exposure. The UV exposure system emitted an average of $5122 \mathrm{~mJ} / \mathrm{cm}^{2}$ per hour of UV-A and $8553 \mathrm{~mJ} / \mathrm{cm}^{2}$ per hour of UVV, which roughly translates to 7200 hours of sunlight exposure. All exposure readings were taken using an EIT Instrument PowerPuck-II UV radiometer. To correlate the natural and artificial UV conditions, several measurements were taken within the exposure system and from direct sunlight as various times of the year. Each sunlight measurement was taken in direct sunlight during clear or partly cloudy day in Northridge, California at $34^{\circ} \mathrm{N}$ and $118^{\circ} \mathrm{W}$. The results are presented in exposure weeks to allow interpretation of the results reported herein independent of any geographical location or climate conditions.

Fig. 1a shows the experimental setup used to measure longitudinal wave (p-wave) speed and corresponding attenuation. Two single element ultrasonic piezoelectric contact transducers (Olympus V133-RM) were used in pitch-catch configuration, with a nominal diameter of 0.25 inches and a central frequency of $2.25 \mathrm{MHz}$. The transducers were powered by square wave pulser (Panametrics $5077 \mathrm{PR}$ ) and were kept in place by securing them to stainless steel holders that also provided constant pressure on the samples, where the weight of the top portion of the holder was $0.62 \mathrm{~kg}$. A small amount of ultrasonic coupler (Olympus U8770023) was applied to each transducer before each measurement. The resulting waveforms were captured and recorded on a digital oscilloscope (Tektronix DPO 2012). The measurements were obtained in two steps. In Step 1, a baseline waveform was recorded with the reference sample of polyurea in between the transducers. In Step 2, the sample of interest (i.e., exposed or unexposed polyurea samples) was then inserted in between the receiving transducer and the reference sample while the 
resulting waveform was recorded. The delay time $\boldsymbol{t}_{\boldsymbol{1}}$ between the waveforms from Step 1 and Step 2 was measured and the p-wave speed was then calculated by dividing the thickness $\boldsymbol{d}$ of each sample by the corresponding delay time (Fig. 2). Additionally, the amplitude of the reference waveform $\boldsymbol{A}_{\boldsymbol{o}}$ (Step 1) and sample waveform $\boldsymbol{A}_{\boldsymbol{I}}$ (Step 2) were measured and used to calculate the p-wave attenuation coefficient $(\alpha)$ using Equation 1 (Qiao, et al., 2011; Graff, 1975).

$$
\alpha=\frac{1}{d} \ln \frac{A_{o}}{A_{1}}
$$

A similar experimental setup was used to measure the shear wave (s-wave) speed, in which a pair of contact shear transducers (Olympus V154-RM) was used with nominal element size of 0.5 inches and central frequency of $2.25 \mathrm{MHz}$. During the s-wave measurement, a different coupler (Olympus SWC) was used. Another important difference between the s-wave (Fig. 1b) and p-wave (Fig. 1a) setups is the replacement of reference sample by an aluminum piece to eliminate the bulk wave that accompanies the generated shear wave. The thickness of the aluminum piece was selected to be 2 inches to avoid interference between shear and bulk waves (Cunningham, J. R., \& Ivey, 1956). The diameter of the aluminum piece was 3 inches to avoid interference from side reflections (Cunningham, J. R., \& Ivey, 1956). The size of the aluminum piece closely matched the experimental setup by Qiao et al. (Qiao, et al., 2011). The remaining steps of the experimental procedure discussed in the previous section were followed to find the shear wave speed and corresponding attenuation coefficient.

The longitudinal $\left(c_{1}\right)$ and the shear $\left(c_{2}\right)$ wave speeds were used to calculate the Poisson's ratio (v) using Equation 2.

$$
v=0.5\left[\frac{c_{1}^{2}-2 c_{2}^{2}}{c_{1}^{2}-c_{2}^{2}}\right]
$$

Subsequently, the effective elastic (E) and effective shear (G) moduli were calculated using pwave, s-wave speeds, Equations 3, and 4, respectively. The density of polyurea ( $\rho$ ) was found to be $1132 \mathrm{~kg} / \mathrm{m}^{3}$. 


$$
\begin{gathered}
E=c_{1}{ }^{2} \rho(1+v)\left(\frac{1-2 v}{1-v}\right) \\
G=c_{2}{ }^{2} \rho
\end{gathered}
$$

The acoustic properties were measured at two different temperatures (i.e. $23^{\circ} \mathrm{C}$ and $40^{\circ} \mathrm{C}$ ) to quantify the effect of temperature as well as UV exposure duration on the measured properties. The entire setup shown in Figures 1a and $1 \mathrm{~b}$ were placed in an oven to keep the temperature constant at $40 \pm 1^{\circ} \mathrm{C}$. It is important to note that the reported properties herein are 'effective' since the overall time delay of the propagating ultrasound wave, i.e., time of flight, is due to the delay of the propagation in the degraded surface layers and sample core.

\section{Results and discussion}

Since the apparent energy of polyurea $(6.25-11.52 \mathrm{kcal} / \mathrm{mol})$ is below the energy associated with ultraviolet $(77-97 \mathrm{kcal} / \mathrm{mol})$, the change in the properties can be attributed to photo-degradation and photo-oxidation (Youssef, 2011; Kockott, 1989). In what follows, UV-induced crazing is presented first and the effect of extended exposure to UV radiation on the change in the ultrasonic properties is discussed thereafter.

\subsection{Crazing}

Crazing was only observed on the side of the samples that was facing the ultraviolet radiation and was limited to only few micrometers in depth, i.e. the depth of the degraded layer. Crazing was witnessed in samples with exposure duration of $6,9,12$, and 15 weeks, while the unexposed samples did not exhibit any crazing. The surface of 3 weeks samples shows the initial stages of crack nucleation due to the surface shrinkage that was induced by ultraviolet radiation. Figure 3 shows the progression of crazing patterns of the third week samples up to the fifteenth week samples where the surface crack size and depth increased from small, discontinuous and shallow cracks to continuous across the width and deep cracks, respectively. For example, Figure 3.a, i.e. surface micrograph of 3 weeks, shows high density of small, shallow cracks with crack openings under $\sim 0.050 \mu \mathrm{m}$. On the other hand, the 15 weeks sample's surface shown in Figure 3.e shows a crack opening of $\sim 0.9 \mu \mathrm{m}$. The onset of noticeable changes to the microstructure of polyurea, and hence crazing, was found to be more than 3 weeks of continuous exposure at the energy levels 
discussed in the previous section. This is attributed to the diffusion properties of polyurea when exposed to ultraviolet radiation at atmospheric conditions. That is, as the exposure duration increases, the depth of the degraded layer increases due to the effect of oxygen reaction-diffusion at the surface promoted by ultraviolet radiation energy or by photo-degradation based on extended ultraviolet exposure.

Generally, the ultraviolet radiation induces three regions in the material: a highly degraded top layer, an interim layer, and an un-radiated core. The highly degraded layer is responsible for the notable color change from transparent yellow in unexposed samples to opaque light brown in the 15 -week exposed samples. This layer also has a higher local stiffness than the un-radiated core (Gigliotti et al. 2011; Olivier et al. 2008). This is consistent with the thermo-oxidative behavior of PMR-15 reported by Tandon et al. when the samples were held isothermally at high temperature (Tandon et al. 2008; Pochiraju \& Tandon 2009; Pochiraju et al. 2008). In the case of polyurea, further in-depth chemical analysis of the top layer is needed to determine whether the degradation is due to oxidation, photo-degradation, or a combination of both.

Therefore, when deploying as-cast polyurea without any pigments in an ultraviolet intensive environment, the samples will show signs of accelerated aging such as discoloration and UV-induced crazing. However, upon loading, catastrophic failure is expected based on the propagation of these cracks. This will in turn compromise the integrity of the structure. The development of crazing after ultraviolet exposure is especially significant for repeated loading scenarios as well as blast impact loading scenarios, where crack propagation will be accelerated.

\subsection{Acoustic Properties}

The effective elastic and shear moduli were calculated from the p-wave and the s-wave speeds, respectively, using Equations 3-4 and the results, along with the corresponding attenuation coefficients, are presented in Figure 4. The effective elastic and shear moduli are reported rather than the wave speeds to account for the slight change ( $1 \%$ overall change) in the Poisson's ratio, which was calculated using Equation 2. Figure 4a shows the change in the effective elastic modulus at $23^{\circ} \mathrm{C}$ and $40^{\circ} \mathrm{C}$ over 15 weeks of continuous exposure to ultraviolet radiation. It is 
noted that the effective elastic modulus at $23^{\circ} \mathrm{C}$ increased slightly from $0.55 \mathrm{GPa}$ for unexposed samples to $0.58 \mathrm{GPa}$ after only three weeks of continuous exposure, which was followed by linearly decreasing trend to reach a value of $0.49 \mathrm{GPa}$ after 15 weeks of UV exposure. The initial increase in the elastic modulus is due to the formation of a semi-continuous degraded layer and prior to crack growth towards the depth of the samples. Once crack growth commences after 3 weeks, the decreasing trend in the elastic modulus is noted. Based on the micrographs presented in Figure 3, crazing can be viewed as a two successive step process, which contributes to the observed behavior of the effective elastic modulus. Initiation first takes place by creating a cluster of shallow surface cracks (Figure 3.a) due to the increase in surface tension. Here, the surface tension can be considered as a bi-axial state of stress acting on the degraded surface layer, which in turn results in a shear stress that conceives the cracks. This is followed by crack growth by the mean normal stress component perpendicular to the crack surface. In short, the loss in stiffness is due to two-step crazing process, which is dependent on the chemical degradation of the surface layer.

Figure 4a also shows the same trend is observed when the elastic modulus is measured at higher temperature $\left(40^{\circ} \mathrm{C}\right)$; however, the magnitudes are smaller in general. The decrease in the modulus measured at $40^{\circ} \mathrm{C}$ from that measured at $23^{\circ} \mathrm{C}$ is expected because the glass transition temperature of polyurea is $-49^{\circ} \mathrm{C}$ (Zhao, Knauss, \& Ravichandran, 2007; Knauss, \& Zhao, 2007). Figure $4 \mathrm{~b}$ displays similar behavior of the shear modulus measured at $23^{\circ} \mathrm{C}$ and $40^{\circ} \mathrm{C}$, where the shear modulus increases slightly over the first three weeks then decreases linearly as the exposure duration increases. The reported values of the elastic moduli herein are relatively higher compared to those reported by $\mathrm{Li}$ et al. of $70 \mathrm{MPa}$ due to the difference in the measurement techniques (Li \& Lua, 2009). In the present study, the moduli were deduced from measurements using the ultrasound technique, while Li et al. tested as-cast polyurea in quasistatic tensile loading conditions using a standard load frame. That is, the ultrasound technique is considered a high strain rate measurement, where polymers are expected to behave stiffer than when tested at low or quasi-static loading rates. When combined with extended UV exposure, the increase in strain rate loading yielded a decrease in the elastic modulus over the exposure period. This, in turn, can compromise structures protected by polyurea-based armors that are subjected to extended UV exposure as well as blast loading scenarios. 
Figure $4 \mathrm{c}$ and $4 \mathrm{~d}$ show the effect of extended UV exposure on the wave attenuation coefficients of polyurea. Figure $4 \mathrm{c}$ first displays that a change in temperature by a mere 17 degrees has a significant effect on the attenuation characteristics of polyurea, where the p-wave attenuation coefficient decreased by $\sim 81 \%$. Interestingly, extended UV exposure did not have any measureable change on the p-wave attenuation properties. However, this is not the case for s-wave attenuation (Fig. 4d), where the same increase in temperature (i.e., 17 degrees) translates to only $34 \%$ drop in the shear wave attenuation of polyurea. In general, the drop in attenuation (p-wave or s-wave) is expected with an increase in temperature since the peak attenuation for polymers usually at or near $\mathrm{T}_{\mathrm{g}}$ depends on the frequency (Qiao, et al., 2011; Mark, 2007; Ivey, Mrowca, \& Guth, 2004). Figure 4d also shows that as the exposure duration increases, the swave attenuation at $23^{\circ} \mathrm{C}$ decreases while attenuation at $40^{\circ} \mathrm{C}$ increases, where both behavior noted to be monotonic. If the UV exposure continues, it is expected that the shear attenuation properties will be insensitive to change in temperature due to the excessive degradation of polyurea and the assumption of monotonic behavior of the s-wave attenuation coefficient continues. From Figures $4 \mathrm{c}$ and $4 \mathrm{~d}$, it also must be noted that the values of the s-wave attenuation coefficient are $40 \%$ and $400 \%$ higher than p-wave attenuation when measured at $23^{\circ} \mathrm{C}$ and $40^{\circ} \mathrm{C}$, respectively. The difference indicates that the main wave attenuation mechanism in polyurea is that of shear. Gupta et al. reported on the exceptional impact mitigation of polyurea, which can be further explained in light of Figure 4 (Gupta, \& Youssef, 2014). That is to say, the impact mitigation of polyurea, i.e. as-cast and UV-exposed, can be divided into an energy absorbance mechanism due to the p-wave attenuation and an energy shunting mechanism due to the shear attenuation. The contribution of the latter is the major impact mitigation mechanism especially as the temperature increases. Additionally, the implantation of polyurea layers into armors has been reported to change the failure from fragments into plugs by Barsoum et al, which also can be explained by the dominance of shear attenuation over p-wave attenuation (Barsoum, G.S. \& Dudt, P.J., 2010). In other words, upon impact loading of polyurea-based armor, the shear attenuation distributes the energy away from the impact site reducing the amount of remaining energy, which in turns causes excessive plastic deformation without reaching the limit of ultimate failure or fragmentation. 
Therefore, the results of the effect of extended UV exposure on the acoustic properties of polyurea is vital to the design of polyurea-based armors and structures in general. The extended exposure decreases the resistance to small strains in high strain rate scenarios. It also has an effect on the attenuation characteristics of polyurea, which can affect the impact mitigation mechanism and in turn can comprise the structural integrity or safety of assets and lives. One design application of the data presented herein is sizing the dimension of a load-bearing member based on a given loading scenario, e.g. bending, and the desired exposure duration. Using basic

mechanics of materials for a simply-supported beam, where maximum deflection is $\frac{-F l^{3}}{48 E I}(F$ is the applied force, $l$ is the beam span, $E$ is the elastic modulus and $I$ is the moment of inertia), in conjunction with data given in Figure 4a, the designer can find that the maximum deflection after 15 -weeks is approximately $20 \%$ higher than deflection after 3 -weeks of exposure. Therefore, the beam dimension needs to be adjusted accordingly.

\section{Conclusion}

In conclusion, the effect of extended ultraviolet exposure on the ultrasonic properties was shown to depend on the exposure duration, temperature as well as the strain rate. In the ultrasound measurements, i.e. high strain rate, the modulus of elasticity was calculated based on the wave speeds and thus reflects the effects of the increase in the strain rate and extended UV exposure. The acoustic wave speeds and p-wave attenuation were found to exhibit minimal change, while shear attenuation showed convergence as the temperature and ultraviolet exposure duration increased. The results can be used in the design of polyurea-based armor systems to determine the long-term reliability as well the effect of extended sun exposure on structure integrity. The results reported herein provide valuable insights into the environmental effects on polyurea-coated structures. The experimental protocol employed in this study is general and applicable to other polymeric materials. Finally, in-depth chemical analyses are required to uncover the change in the molecular structure of polyurea as exposure duration increases.

\section{Acknowledgment}

The research leading to these results was supported in part by the United States Department of Defense under Grant Agreement No. W911NF-14-1-0039. 


\section{References}

Amini, M. R., Isaacs, J. B., \& Nemat-Nasser, S.: Experimental investigation of response of monolithic and bilayer plates to impulsive loads. International Journal of Impact Engineering, 37(1), 82-89 (2010)

Amirkhizi, A. V., Isaacs, J., McGee, J., Nemat-Nasser, S.: An experimentally-based viscoelastic constitutive model for polyurea, including pressure and temperature effects. Philosophical Magazine. 86, 5847-5866 (2006)

Bond, R., Morton, G. F., \& Krol, L. H.: A tailor-made polymer for tyre applications. Polymer, 25(1), 132-140 (1984)

Barsoum, G.S., Dudt, P.J.: The fascinating behaviors of ordinary materials under dynamic conditions. Ammtiac Quarterly 4, 11-14 (2010)

Brinson, J.: Ultra-violet radiation effect on the mechanical properties of polyurea. Unpublished master's thesis. CSUN, Northridge (2013)

Choi, I. S., Roland, C. M., \& Bissonnette, L. C.: An elastomeric ejection system. Rubber Chemistry and Technology, 67(5), 892-903 (1994)

Cunningham, J. R., \& Ivey, D. G.: Dynamic properties of various rubbers at high frequencies. Journal of Applied Physics, 27(9), 967-974 (1956)

Davidson, J. S., Porter, J. R., Dinan, R. J., Hammons, M. I., \& Connell, J. D.: Explosive testing of polymer retrofit masonry walls. Journal of Performance of Constructed Facilities, 18(2), 100106 (2004)

Gigliotti, M., Grandidier, J.C., \& Lafarie-Frenot, M.C.: Assessment of chemo-mechanical couplings in polymer matrix materials exposed to thermo-oxidative environments at high temperatures and under tensile loadings. Mechanics of Materials, 43.8, 431-443 (2011).

Graff, K. F.: Wave motion in elastic solids. Courier Dover Publications (1975)

Gupta, V., \& Youssef, G.: Orientation-dependent impact behavior of polymer/EVA bilayer specimens at long wavelengths. Experimental Mechanics, 1-5 (2014)

Heinrich, G., \& Kaliske, M.: Theoretical and numerical formulation of a molecular based constitutive tube-model of rubber elasticity. Computational and Theoretical Polymer Science, 7(3), 227-241 (1997)

Hoppie, L. O.: The use of elastomers in regenerative braking systems. Rubber Chemistry and Technology, 55(1), 219-232 (1982) 
Ivey, D. G., Mrowca, B. A., \& Guth, E.: Propagation of ultrasonic bulk waves in high polymers. Journal of Applied Physics, 20(6), 486-492 (2004)

Jain, A.: Strength/moisture relationship for interfaces and joints for robust prediction of reliability. Unpublished doctoral dissertation. UCLA, Los Angeles (2007)

Jain, A., \& Gupta, V.: Construction and characterization of stainless steel/polyurea/E-glass composite joints. Mechanics of Materials, 46, 16-22 (2012)

Jain, A., Gupta, V., \& Basu, S. N.: A quantitative study of moisture adsorption in polyimide and its effect on the strength of the polyimide/silicon nitride interface. Acta materialia, 53(11), 31473153 (2005)

Jain, A., Youssef, G., \& Gupta, V.: Dynamic tensile strength of polyurea-bonded steel/E-glass composite joints. Journal of Adhesion Science and Technology, 27(4), 403-412 (2013)

Jiao, T., Clifton, R. J., \& Grunschel, S. E.: High strain rate response of an elastomer. In Shock compression of condensed matter-2005: Proceedings of the Conference of the American Physical Society Topical Group on Shock Compression of Condensed Matter (Vol. 845, No. 1, pp. 809812). AIP Publishing (2006)

Kim, H.: In-situ measurement of intrinsic interface strength and moisture-effected interfacial fracture energy. Ph.D. Dissertation, Department of Mechanical and Aerospace Engineering, UCLA, Los Angeles, CA (2008)

Kim, H., Citron, J., Youssef, G., Navarro, A., \& Gupta, V.: Dynamic fracture energy of polyurea-bonded steel/E-glass composite joints. Mechanics of Materials, 45, 10-19 (2012)

Klüppel, M., \& Heinrich, G.: Rubber friction on self-affine road tracks. Rubber Chemistry and Technology, 73(4), 578-606 (2000)

Knauss, W. G., \& Zhao, J.: Improved relaxation time coverage in ramp-strain histories. Mechanics of Time-Dependent Materials, 11(3-4), 199-216 (2007)

Kockott, D.: Natural and artificial weathering of polymers. Polymer Degradation and Stability, 25(2), 181-208 (1989)

Li, C. \& Lua J.: A hyper-viscoelastic constitutive model for polyurea. Materials letters, 63(11), 877-880 (2009).

Mark, J. E. (Ed.): Physical properties of polymers handbook (pp. 453-557). New York: Springer (2007)

Olivier, L., Ho, N. Q., Grandidier, J. C., \& Lafarie-Frenot, M. C.: Characterization by ultramicro indentation of an oxidized epoxy polymer: Correlation with the predictions of a kinetic model of oxidation. Polymer Degradation and Stability, 93(2), 489-497, (2008). 
Persson, B. N., Tartaglino, U., Albohr, O., \& Tosatti, E.: Rubber friction on wet and dry road surfaces: The sealing effect. Physical Review B, 71(3), 035428 (2005)

Pochiraju, K., \& Tandon, G. P.: Interaction of oxidation and damage in high temperature polymeric matrix composites. Composites Part A: Applied Science and Manufacturing, 40(12), 1931-1940 (2009)

Pochiraju, K. V., Tandon, G. P., \& Schoeppner, G. A.: Evolution of stress and deformations in high-temperature polymer matrix composites during thermo-oxidative aging. Mechanics of TimeDependent Materials, 12(1), 45-68 (2008).

Pochiraju, K. V., Tandon, G. P., \& Schoeppner, G. A.: Evolution of stress and deformations in high-temperature polymer matrix composites during thermo-oxidative aging. Mechanics of Time-Dependent Materials, 12(1), 45-68 (2008).

Qiao, J., Amirkhizi, A. V., Schaaf, K., Nemat-Nasser, S., \& Wu, G.: Dynamic mechanical and ultrasonic properties of polyurea. Mechanics of Materials, 43(10), 598-607 (2011)

Rahalkar, R. R.: Dependence of wet skid resistance upon the entanglement density and chain mobility according to the Rouse theory of viscoelasticity. Rubber Chemistry and Technology, 62(2), 246-271 (1989)

Roland, C. M., Twigg, J. N., Vu, Y., \& Mott, P. H.: High strain rate mechanical behavior of polyurea. Polymer, 48(2), 574-578 (2007)

Sarva, S. S., Deschanel, S., Boyce, M. C., \& Chen, W.: Stress-strain behavior of a polyurea and a polyurethane from low to high strain rates. Polymer, 48(8), 2208-2213 (2007)

Youssef, G. H.: Dynamic properties of Polyurea. Unpublished Doctoral Thesis. UCLA, Los Angeles (2011)

Youssef, G., \& Gupta, V.: Dynamic response of polyurea subjected to nanosecond rise-time stress waves. Mechanics of Time-Dependent Materials, 16(3), 317-328 (2012)

Youssef, G., \& Gupta, V.: Resonance in Polyurea-Based Multilayer Structures Subjected to Laser-Generated stress waves. Experimental Mechanics, 53(2), 145-154 (2013)

Zhao, J., Knauss, W. G., \& Ravichandran, G.: Applicability of the time-temperature superposition principle in modeling dynamic response of a polyurea. Mechanics of TimeDependent Materials, 11(3-4), 289-308 (2007)

Zhao, J., Knauss, W. G., \& Ravichandran, G.: A new shear-compression-specimen for determining quasistatic and dynamic polymer properties. Experimental Mechanics, 49(3), 427 436 (2009) 


\section{List of Figures}

Figure 1. Experimental setup to measure (a) longitudinal and (b) shear wave speeds and attenuations.

Figure 2. Illustration of reference (solid-line) and sample (dash-line) waveforms.

Figure 3. Crazing progression as function of UV exposure time.

Figure 4. Effect of extended exposure of ultraviolet on (1) elastic modulus, (b) shear modulus, (c) p-wave attenuation, and (d) s-wave attenuation. 


\section{FIGURES}

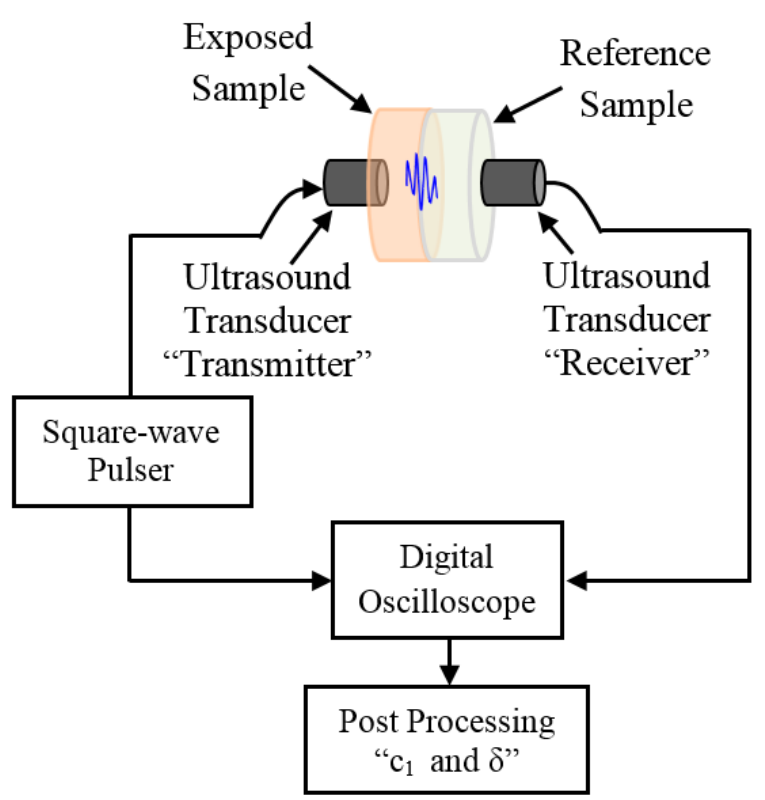

(a)

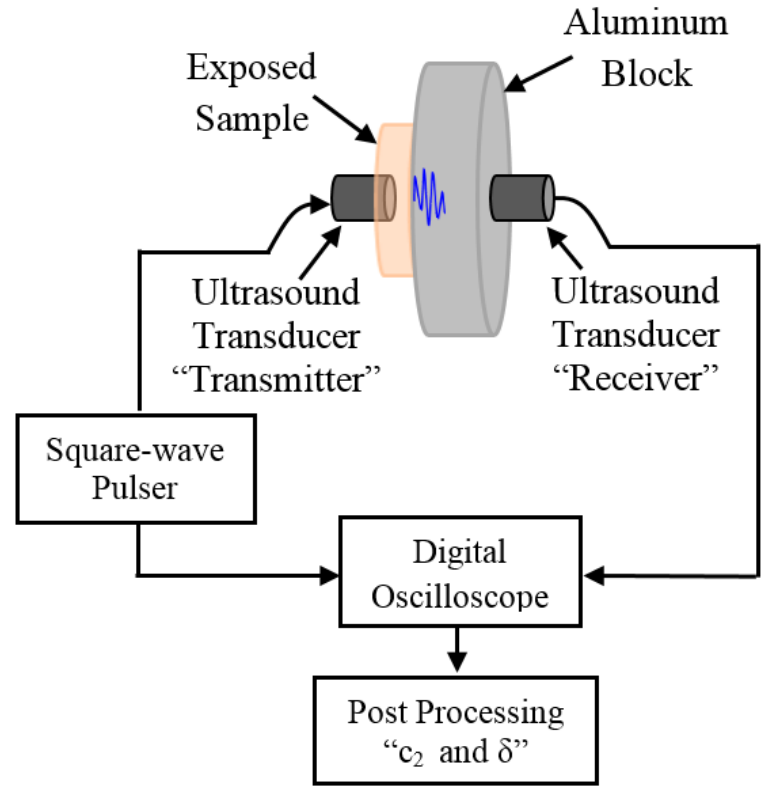

(b)

Figure 1. Experimental setup to measure (a) longitudinal and (b) shear wave speeds and attenuations. 


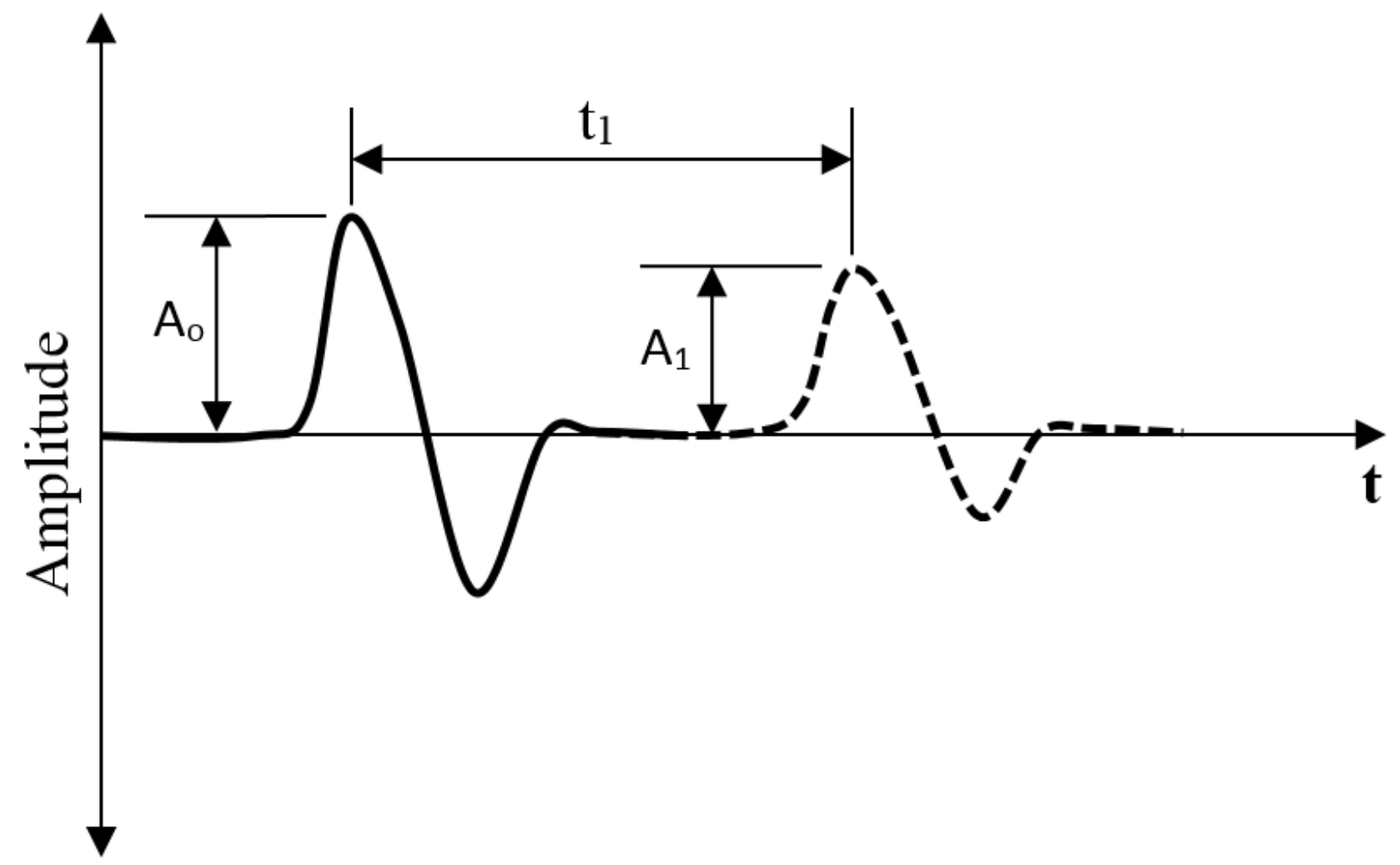

Figure 2. Illustration of reference (solid-line) and sample (dash-line) waveforms. 

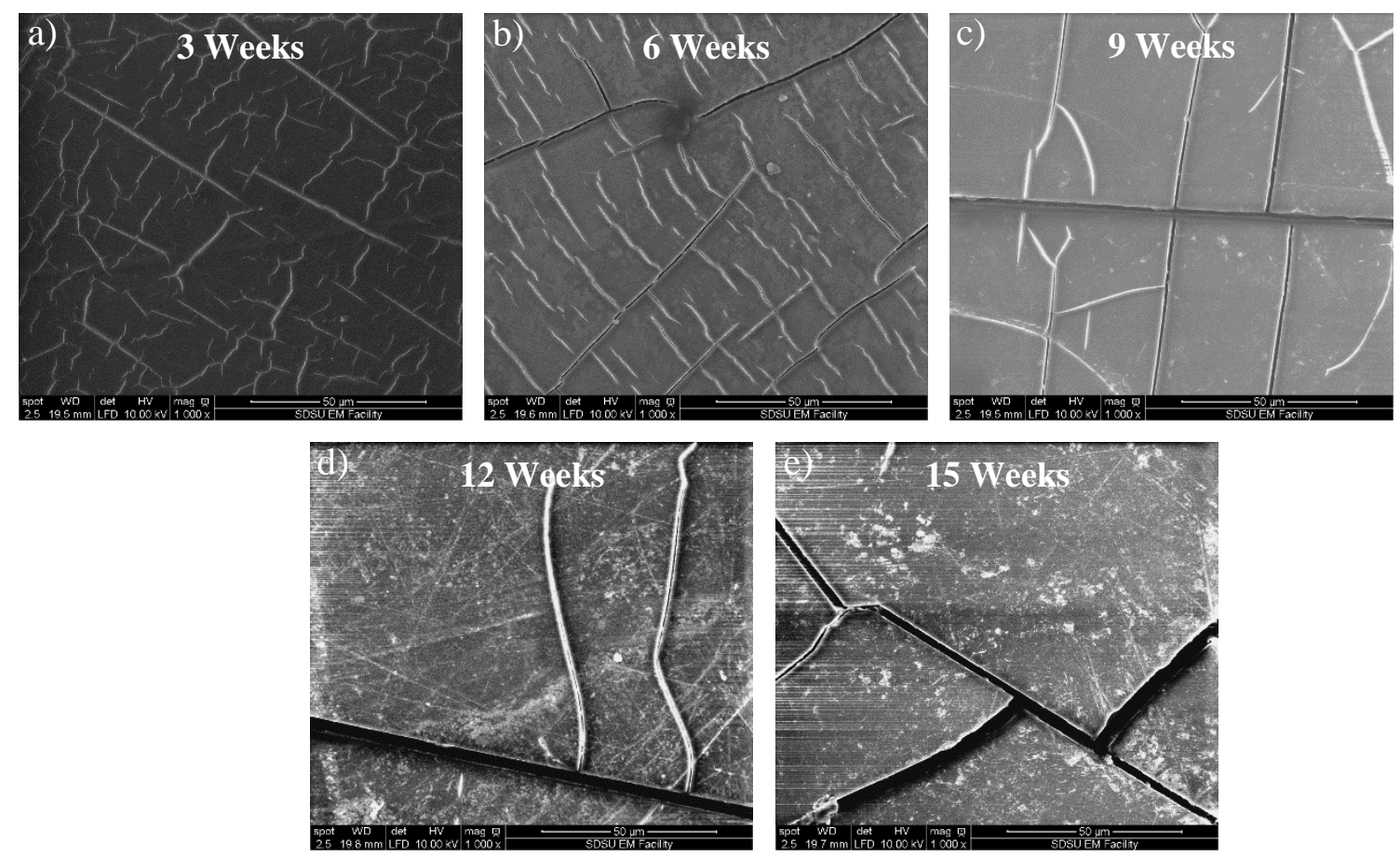

Figure 3. Crazing progression as function of UV exposure time. 

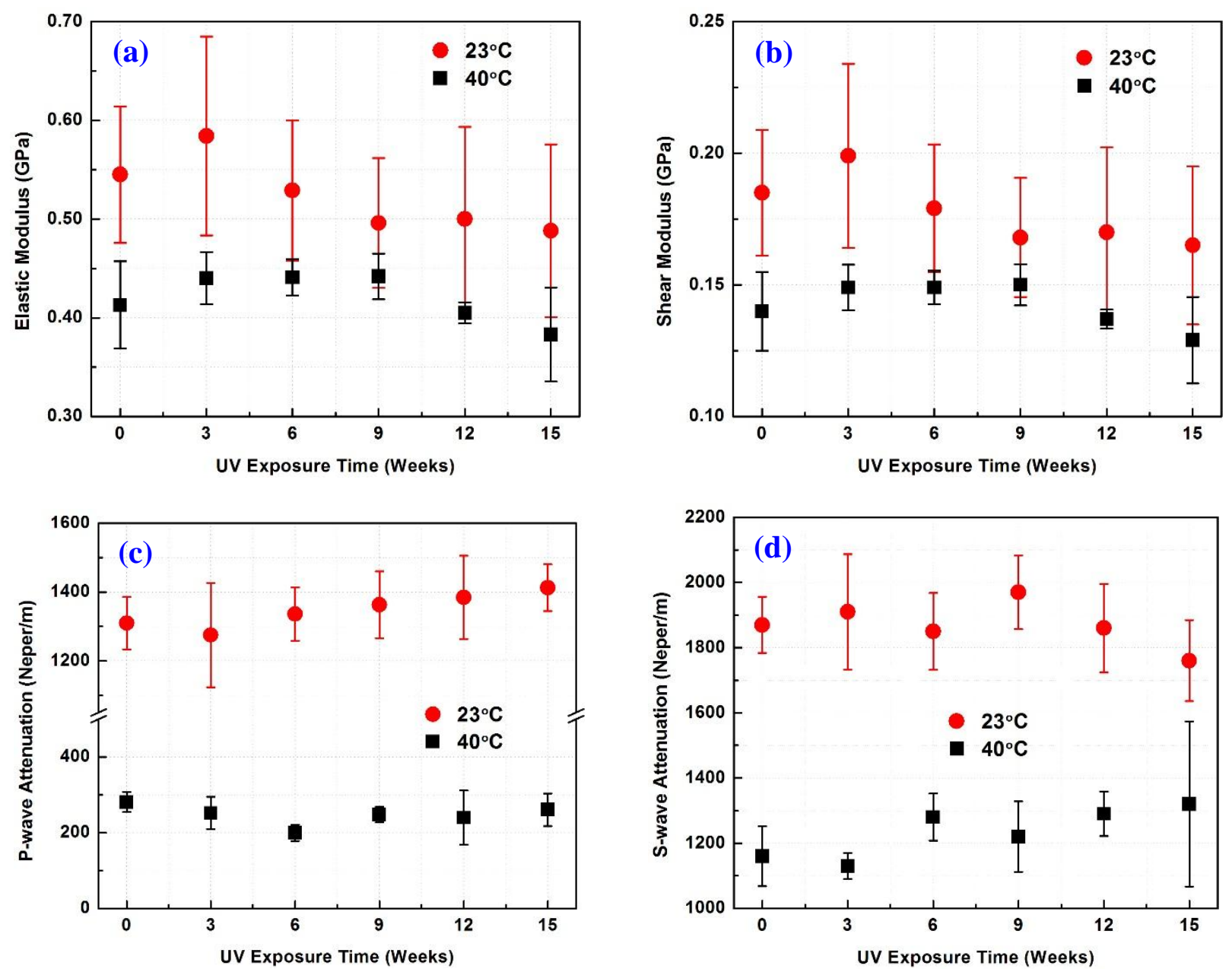

Figure 4. Effect of extended exposure of ultraviolet on (a) elastic modulus, (b) shear modulus, (c) p-wave attenuation, and (d) s-wave attenuation. 